Pure \& App Z. Chem., Vo1. 56, No. 3, pp. 447-460, 1984. Printed in Great Britain.
$0033-4545 / 84 \$ 3.00+0.00$

Pergamon Press Ltd. (1)1984 IUPAC

\title{
QUANTITATIVE STUDIES OF ELECTRON-TRANSFER INITIATION OF POLYMERIZATION
}

\section{Michael Szwarc}

Department of Chemistry (B-014), University of California San Diego, La Jolla, CA 92093

Abstract - Electron-transfer initiation of anionic polymerization involves two step's: electron-transfer from a donor to a monomer acceptor, jielding monomeric radicalanions followed by dimerization of the latter. Dimerization yields dimeric dianions and the respective carbanions propagate the polymerization. Reaction of the monomeric radical-anions with unreduced monomer was shown to be of little significance.

It was shown that the electron-transfer equilibrium is attained in an extremely short time and the dimerization is the rate determining step of initiation. The equilibrium transfer was determined. For conventional homogeneous donors, e.g., sodium naphthalenide or biphenylide, the equilibrium is unfavorable in respect to monomer, even when it is in large excess.

The rate of the dimerization was measured, and it was shown to depend on the nature of counter-ions. The numerical results are discussed and a tentative explanation is proposed for the findings.

Heterogeneous electron-transfer initiation is discussed with emphasis on the adsorption and desorption processes. The results of some copolymerization are rationalized.

\section{INTRODUCTION}

Vinyl, vinylidene or diene polymerization is usually initiated by addition of some moiety, $X$, to a monomer, $\mathbb{M}$, leading to the formation of a reactive end-group capable of sustaining propagation. The ensuing polymerization is propagated through a radical, cationic, or anionic mechanism, depending upon whether $\mathrm{X}$ is a radical, a cation, or an anion. This is shown below:

$$
\begin{aligned}
& \text { X. + C:C X.C.C. , radical polymerization } \\
& \mathrm{X}^{+}+\mathrm{C}: \mathrm{C} \text { X.C.C. }{ }^{+} \text {, cationic polymerization } \\
& \mathrm{X}^{-}+\mathrm{C}: \mathrm{C} \text { X.C. } \mathrm{C}^{-} \text {, anionic polymerization, }
\end{aligned}
$$

and in each case while one end of a growing polymer is active, the other remains inert. 
An entirely different mechanism of initiation was proposed in the $1950^{\prime} \mathrm{s}$ (Ref. 1), namely, initiation caused by electron-transfer to monomer. An electron transferred from a suitable donor, $A$ or $A^{-}$, to a monomer, $M$, converts it into a monomeric radical-anion

$$
\left.A\left(\text { or } A^{-}\right)+M^{+}=A^{+} \text {(or } A\right)+M^{-} \quad, K_{t r} \text {. }
$$

The monomeric radical-anions dimerize into dimeric dianions,

$$
\begin{aligned}
& 2 \mathrm{M}^{-} \rightarrow^{-} \mathrm{M}^{-\mathrm{M}^{-}}, \quad \mathrm{k}_{\mathrm{d}} \text {, } \\
& \text { e.g. , } \\
& 2 \mathrm{Ph} . \mathrm{CH}: \mathrm{CH}_{2}=-\mathrm{Ph} \cdot \mathrm{CH} \cdot \mathrm{CH}_{2} \cdot \mathrm{CH}_{2} \cdot \overline{\mathrm{CH}} \cdot \mathrm{Ph} \text {, }
\end{aligned}
$$

or react with the available monomer, forming dimeric radical-anions, $\mathrm{M}-+\mathrm{H}-\mathrm{M} \cdot \mathrm{Wi} \cdot \mathrm{k}_{\mathrm{a}}$, e.g. Ph. $\mathrm{CH}: \mathrm{CH}_{2}-+\mathrm{Ph} \cdot \mathrm{CH}: \mathrm{CH}_{2}-\mathrm{Ph} \cdot \mathrm{CH} \cdot \mathrm{CH}_{2} \cdot \mathrm{CH}_{2} \cdot \dot{\mathrm{CH}} \cdot \mathrm{Ph}$.

The resulting dimeric dianions initiate anionic polymerization propagated from both ends of the macromolecules,

$$
-\mathrm{M}^{\mathrm{M}} \mathrm{M}^{-} \stackrel{\mathrm{N}}{\rightarrow}-\mathrm{N} . \mathrm{M} \text {.......M.M. }
$$

a novel situation leading to valuable consequences. The dimeric radicalanions whenever formed, could perhaps initiate anionic propagation ensuing from one of their ends and simultaneously a radical polymerization from the other. However, this is a highly unlikely event. Most probably, they disproportionate, dimerize, or become reduced to dianions, viz.

or

$$
\begin{aligned}
& 2^{-} \text {M.M. } \rightarrow{ }^{-M} \cdot M^{-}+2 M, \\
& 2^{-} \text {M.M. - }{ }^{-} \text {H.M.M.M-, }
\end{aligned}
$$

$$
-M^{\prime} \cdot M .+A\left(\text { or } A^{-}\right)-M^{-} M^{-}+A^{+}(\text {or } A)
$$

The feasibility of electron-transfer initiation was fully confirmed in subsequent studies. However, its quantitative aspects remained unknown. It is my intention to discuss here this subject and show the methods by which the required information was obtained. In that work flashphotolysis was extensively used, and therefore a few words about the basic feature of this technique are in place. A detailed description of its usage in systems studied by us is given elsewhere (Ref. 2).

\section{Principles of Flash-Photolysis}

The experimental set-up is shown schematically in Fig. 1. The approximately $10^{-6} \mathrm{M}$ solution of the active ingredient is introduced into a cylindrical, $10 \mathrm{~cm}$. long quartz cell with optically flat end-windows. The cell is placed between two parallel flash lamps, being surrounded by cuvets containing a UV absorbing solution. A properly collimated beam of monitoring light that passes through the cell is focused on the slit of monochromator. By appropriate setting of the monochromator, one allows the light of the desired wavelength to reach a photomultiplier, and its output is amplified and fed into an oscilloscope. 


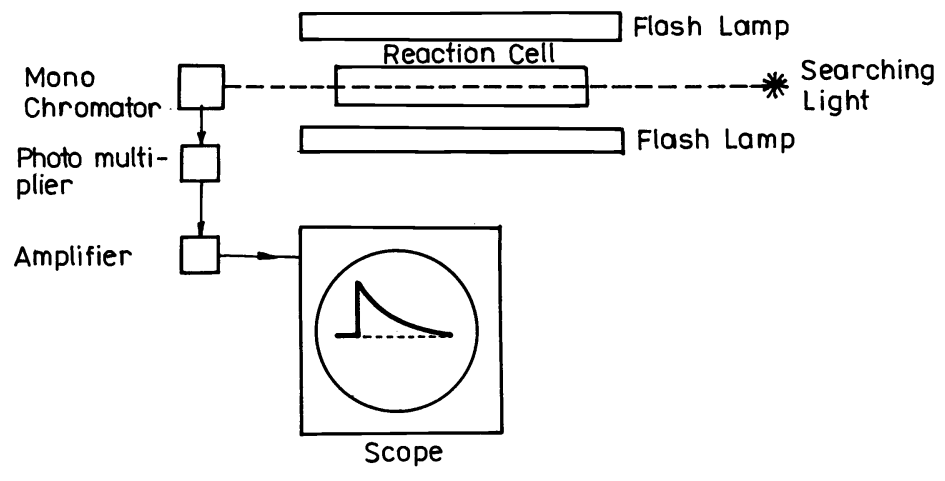

Fig. 1

Triggering the scope, but not the flash lamps, one gets on its screen a horizontal zero-line, showing how much light of a desired wavelength passes through the unphotolyzed solution. Subsequently, the flash lamps are triggered by the scope which has been activated $\sim 100 \mu \mathrm{sec}$. earlier. The ensuing photolysis converts some of the original reagents into transient species, which react during the dark period following a flash and eventually yield the final products of the photolysis. The progress of the dark reaction is revealed by changes in the absorbance of the monitoring light displayed on the oscilloscope's screen. The resulting curve gives the intensity of the transmitted light as a function of time. The systems studied by us were perfectly reversible. At any wavelength the initially enhanced absorbance or bleaching decayed to a zero-line as shown in Fig. 1. This observation implies that the transients formed by photolysis regenerate the original reagents during the dark period of the reaction. Therefore, flashing could be repeated over and over again, leaving unaltered the ultimate composition of the photolyzed solution. By varying the setting of the monochromator, a family of curves is obtained, each depicting the return of the photolyzed solution to its original state but monitored at a different wavelength. Such curves permit one to calculate the optical density of the photolyzed solution at a chosen time, say $100 \mu \mathrm{sec}$. after each flash, and at a desired wavelength. This, in turn, allows construction of a difference spectrum the difference in the absorbance of the transients and the original reagents at that time.

Quantitative Treatment of Homogeneous Electron-Transfer Inltiation

A difference spectrum obtained by flashing a THF solution of dimeric dianions of $\propto$-methyl styrene, $\mathrm{K}^{+}, \mathrm{C}\left(\mathrm{CH}_{3}\right)(\mathrm{Ph}) \cdot \mathrm{CH}_{2} \cdot \mathrm{CH}_{2} \cdot \mathrm{C}\left(\mathrm{CH}_{3}\right)(\mathrm{Ph}), \mathrm{K}^{+}=$ $\mathrm{K}^{+},-\propto . \propto^{-}, \mathrm{K}^{+}$, is shown in Fig. 2(Ref. 3). Although it fades with time, its shape remains unchanged indicating that the transients formed by flash directly regenerate the original dimeric dianions, no other intermediates or products being formed. 


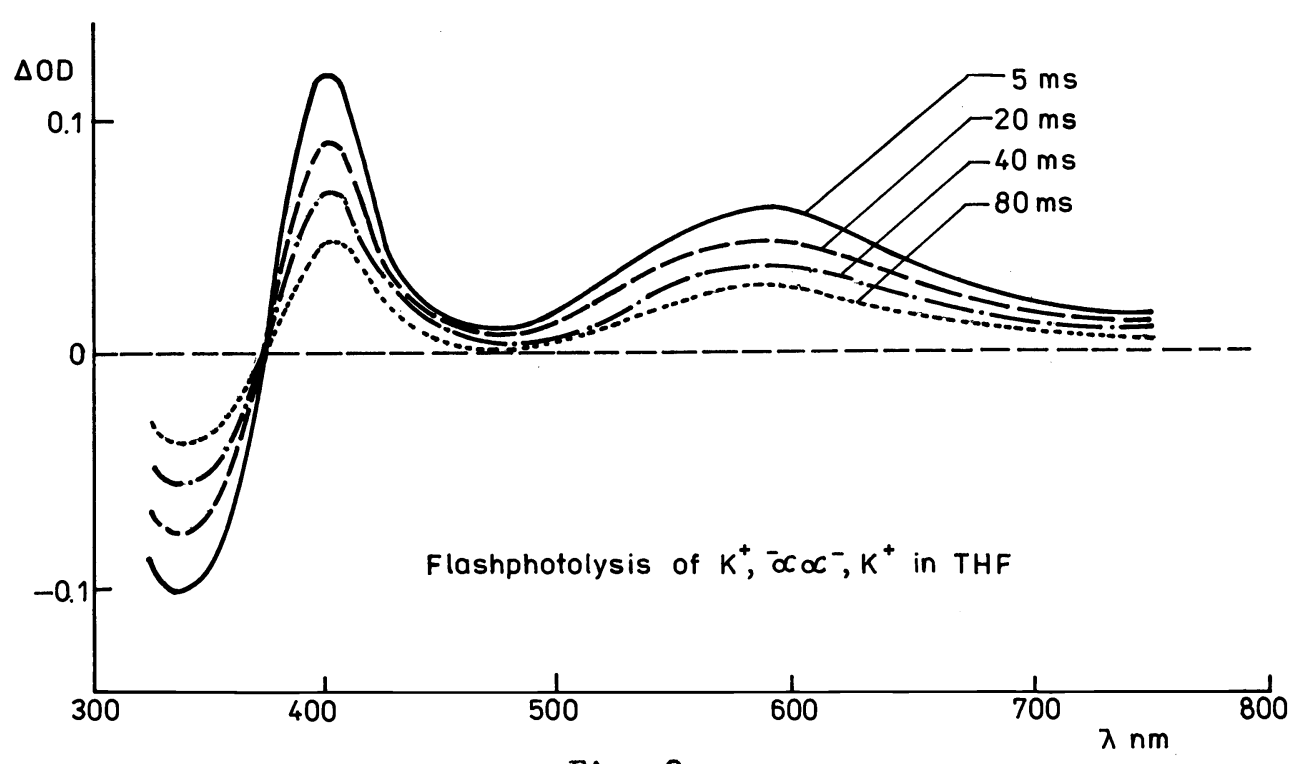

Fig. 2

The difference spectrum results from the absorbance of the transient and bleaching of the dimers. Hence, the spectrum of the transient could be $e$ constructed by adding the known spectrum of the photolyzed dimers to the observed difference spectrum. Such a procedure is illustrated by Fig. 3. The spectrum of the transient agrees with that of $\propto$-methyl styrene radical anions obtained by pulse radiolysis and reported by three independent groups (Ref. 4). One concludes therefore that the dimeric dianions of $\propto$-inethyl styrene have been photo-dissociated by flash into $\propto$-methyl styrene radical-anions, $\propto .^{-}$, i.e.

$$
\mathrm{K}^{+},-\overline{-}^{-}, \mathrm{K}^{+} \stackrel{\mathrm{h} \nu}{\longrightarrow} 2 \mathrm{~K}^{+}, \bar{\infty}_{0},
$$

and in the subsequent dark period they dimerized into the dimers.

Analogous studies of flash photolysis of THF solutions of dimeric dianions of 1,l-diphenyl-ethylene, $\mathrm{Cat}^{+}, \overline{\mathrm{c}}(\mathrm{Ph})_{2} \cdot \mathrm{CH}_{2} \cdot \mathrm{CH}_{2} \overline{\mathrm{C}}(\mathrm{Ph})_{2}$, $\mathrm{Cat}^{+}=\mathrm{Cat}^{+}$, D. $\mathrm{D}^{-}$, Cat ${ }^{+}$, led to the spectrum of the respective transient shown in Fig. 4 (Ref. 5). Its similarity with the spectrum of 1,1-diphenyl-ethylene

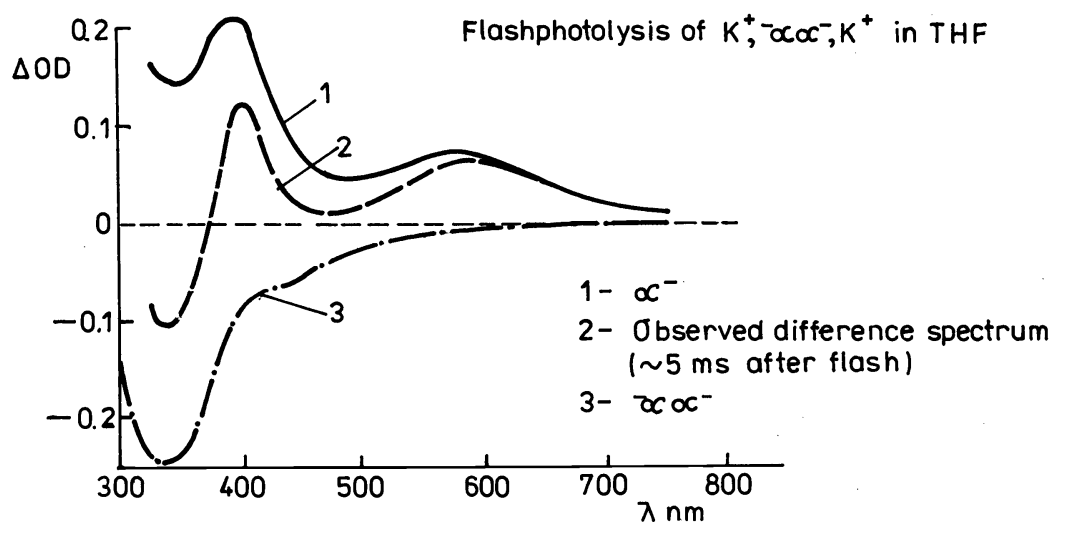




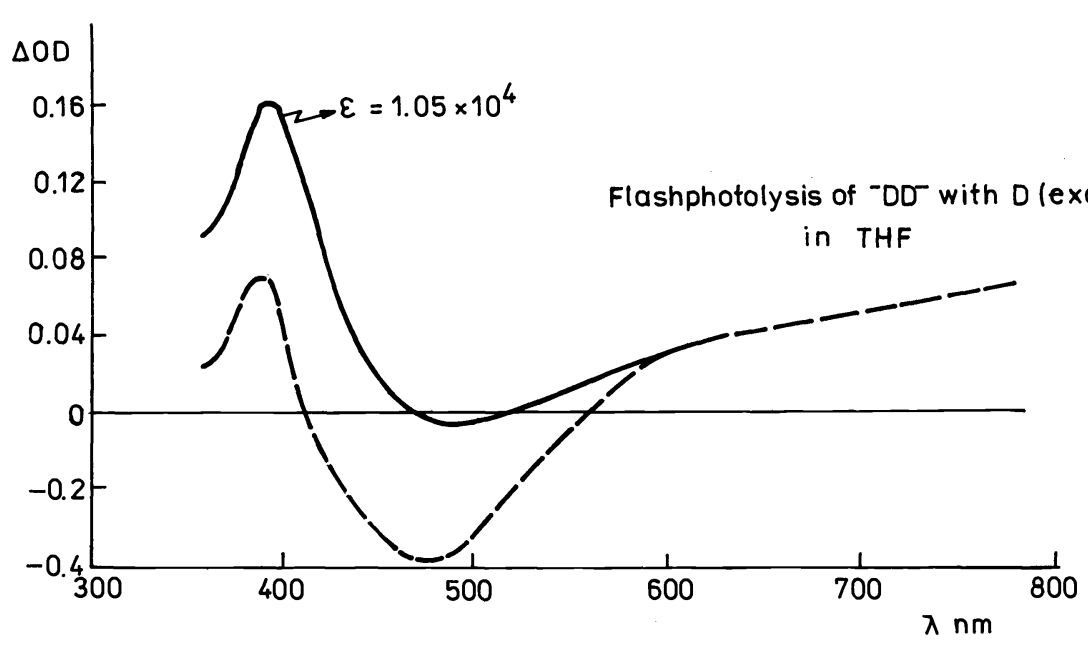

Fig. 4

radical-anions, $D^{-}$, reported by Hamill (Ref. 6) who radiolyzed frozen 2-MeTHF solution of that hydrocarbon, implies that flash-photolysis of those dimeric dianions again leads to their photo-dissociation into the monomeric radical-anions, viz.

$$
\mathrm{Cat}^{+},{ }^{-D . D^{-}}, \mathrm{Cat}^{+} \stackrel{\mathrm{h \nu}}{\longrightarrow} 2 \mathrm{D}^{-}, \mathrm{Cat}^{+}
$$

For both systems reciprocals of $\Delta$ optical density are linear with time.

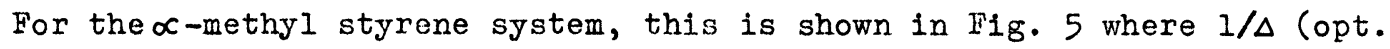
density) at $340 \mathrm{~nm}, 400 \mathrm{~nm}$, and $600 \mathrm{~nm}$ is plotted vs. time, and for the

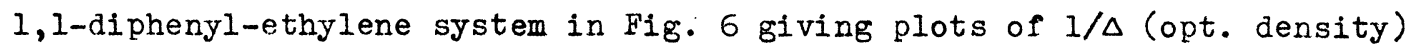
at $390 \mathrm{~nm}, 470$, and $750 \mathrm{~nm}$ vs time. Iinearity of those plots proves the bimolecular character of processes regenerating the dimeric dianions. Slopes of those plots provide, therefore, the values of the respective dimerization constants, $\underline{k}_{d}$, divided by $\varepsilon l$, where $\varepsilon$ is the respective effective molar absorbance and $l$ is the length of the cell. The effective molar absorbances were determined by various methods (Ref. 3,5) and thereafter the dimerization constants of $\mathrm{D}^{-}, \mathrm{Cat}^{+}$and $\mathrm{C}^{-}$, Cat ${ }^{+}$were calculated. Their values, listed in Table $I$, show their dependence on the nature of cation increasing with its radius. Significantly, the dimerization of radical-anions is substantially slower than of small, neutral radicals, the latter being diffusion controlled, i.e., its bimolecular rate constants are $\sim 10^{10} \mathrm{M}^{-1} \mathrm{sec}^{-1}$. Apparently, the repulsion of the negatively charged particles (for free ions) or of the unfavourably oriented dipoles (for lon-pairs) leads to retardation. Surprisingly, $\propto-, \mathrm{Cat}^{+}$dimerize much slower than $\mathrm{D}^{-}, \mathrm{Cat}^{+}$, an unexplained finding.

Since the dimerization is the rate determining step in the initiation of polymerization by aromatic radical-anions, its rate is

$$
\underline{\mathrm{k}}_{\mathrm{d}} \mathrm{K}_{\mathrm{tr}}^{2}\left\{\left[\mathrm{~A} \cdot, \mathrm{Cat}^{+}\right] \cdot[\text { monomer }] /[\mathrm{A}]\right\}^{2}
$$




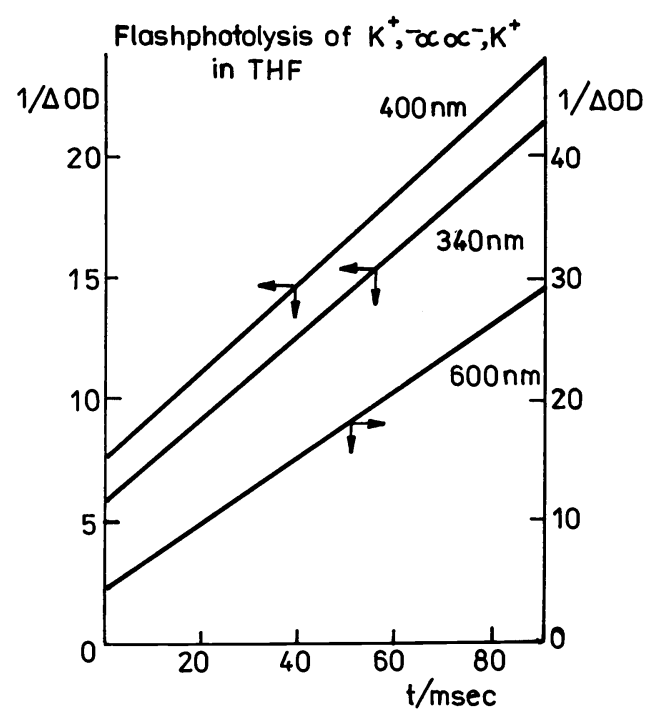

Fig. 5

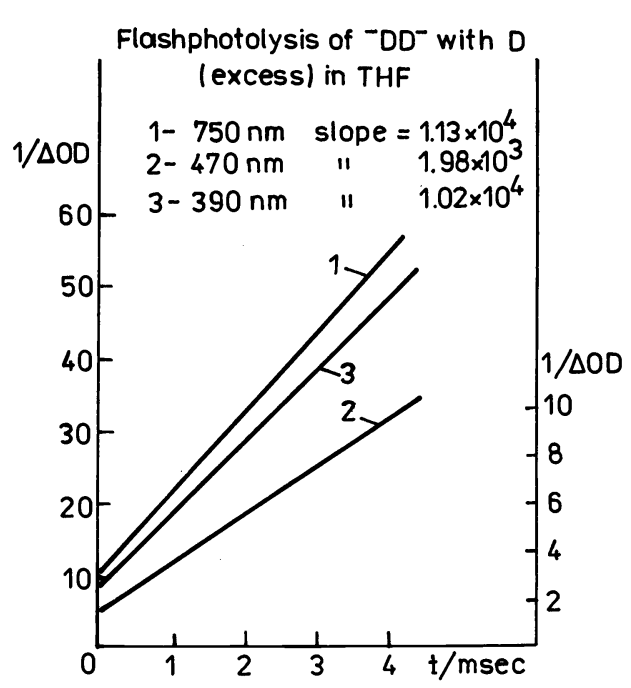

Fig. 6

Here $K_{t r}$ denotes the equilibrium constant of electron-transfer. Because the relaxation time is extremely short, the equilibrium concentration of the monomeric radical-anions is unperturbed by their dimerization. ${ }^{x} A$ numerical example illuminates this point.

Consider the initial conditions of a $1 \mathrm{M} \mathrm{THF}$ solution of a monomer containing $10^{-4} \mathrm{M}$ of an initiator $\mathrm{A}^{-}, \mathrm{Cat}^{+}$. For as $10 \mathrm{w} \mathrm{K}_{\mathrm{tr}}$ as $10^{-5}$, the electron-transfer equilibrium, established within a few $\mu \mathrm{sec}$. , converts $27 \%$ of $\mathrm{A}^{-}, \mathrm{Cat}^{+}$into monomer-, $\mathrm{Cat}^{+}$. It takes less than $0.2 \mathrm{sec}$. to produce $95 \%$ of all the dimeric dianions expected in the quantitative conversion, even for as low $\underline{k}_{d}$ as $10^{6} \mathrm{M}^{-1} \mathrm{sec}^{-1}$. In that time less than 50 monomer molecules are added to each of the formed growing centers, provided the propasation constant is not larger than $250 \mathrm{M}^{-1} \mathrm{sec}^{-1}$ - a rather high value, while at the completion of polymerization $10^{4}$ molecules are added.

The dimerization competes with monomer addition to monomeric radical-anions

$$
M_{0}^{-}+M-\cdot M \cdot M^{-} \quad, \underline{k}_{a}
$$

Most likely $\underline{k}_{a}$ is smaller than the propagation constant, i.e., $\underline{k}_{a}<$ $250 \mathrm{~m}^{-1} \mathrm{sec}^{-1}$. However, since the concentration of the monomer is at least $10^{4}$ times larger than that of the monomeric radical-anions, the

$\mathbf{x}$

This relaxation time is given by $z=1 /\left\{\underline{k}_{f}[M]+\underline{k}_{b}[A]\right\}, \underline{k}_{f}$ and $\underline{k}_{b}$ denoting the forward and backward rate constants of the electron-transfer. Thus, $Z$ is at the most $I \mu \mathrm{sec}$. 
adition competes efficiently with the dimerization. Nevertheless, the resulting dimeric radical-anions, .M.M- play no role in the polymerization because they are destroyed as soon as formed by the diffusion controlled disproportionation (rate constant $\sim 10^{10} \mathrm{M}^{-1} \mathrm{sec}^{-1}$ ). Hence, radical propagation is imperceptible in such systems.

TABIE 1. Dimerization constants of $\mathrm{D}^{-}, \mathrm{Cat}^{+}$and $\alpha^{-}, \mathrm{Cat}^{+}$ in THF at $250 \mathrm{C}$

\begin{tabular}{ccc}
\hline $\mathrm{Cat}^{+}$ & $10^{-8}{\underline{\underline{\mathrm{k}_{\mathrm{d}}}}}^{\mathrm{MSec}}\left(\mathrm{D}^{-}, \mathrm{Cat}^{+}\right)$ & $10^{-7}{\underline{\underline{\mathrm{k}_{\mathrm{d}}}}}_{\mathrm{Msec}}\left(\alpha_{-}^{-}, \mathrm{Cat}^{+}\right)$ \\
\hline $\mathrm{Li}^{+}$ & 1.2 & - \\
$\mathrm{Na}^{+}$ & 3.5 & 0.2 \\
$\mathrm{~K}^{+}$ & 10.0 & $1.0-1.2$ \\
$\mathrm{Cs}^{+}$ & 30.0 & - \\
\hline
\end{tabular}

\section{Dissociation of the dimeric dianions}

The dimeric radical-anions are stable. The rate of dissociation of $\mathrm{K}^{+},{ }^{-} \propto \propto^{-}$, $\mathrm{K}^{+}$was determined by the following procedure (Ref. 7): $\propto$-methyl styrene perdeuterated in phenyl groups, $\propto_{5 D}$, was prepared and converted into dimeric dianions, $\mathrm{K}^{+},{ }^{-} \infty_{5 D} \cdot \infty_{5 D^{-}}, \mathrm{K}^{+}$. Their THF solution was mixed with a solution of ordinary protic dimers, $\mathrm{K}^{+},{ }^{-} \propto, \infty^{-}, \mathrm{K}^{+}$. The reversible dissociation-association

$$
\begin{array}{ll}
\mathrm{m}=238, & \mathrm{~K}^{+},-\propto . \propto^{-}, \mathrm{K}^{+} \stackrel{\underline{\mathrm{k}}_{\text {diss }}}{=} 2 \propto_{\cdot}^{-}, \mathrm{K}^{+} \\
\mathrm{m}=243 & \mathrm{~K}^{+},-\propto_{5 \mathrm{D}}, \mathrm{K}^{+} \underset{\mathrm{k}_{\text {diss }}}{\mathrm{m}=2.48} \mathrm{~K}^{+}, \propto_{5 \mathrm{D}} \cdot \propto_{5 \mathrm{D}}, \mathrm{K}^{+} \rightleftharpoons 2 \propto_{5 \mathrm{D}}^{-}, \mathrm{K}^{+}
\end{array}
$$

forms mixed aimers, $\mathrm{K}^{+},-\infty . \propto_{5 \mathrm{D}}, \mathrm{K}^{+}$. For a $50: 50$ mixture the rate of mixed dimers formation is equal to $1 / 2$ of the rate of the dimer's dissociation, $\mathrm{k}_{\text {diss. }}$. To determine this rate the mixture was kept at a constant temperature and at desired time intervals ( $12 \mathrm{~h}, 24 \mathrm{~h}$, etc.) aliguots were removed and protonated by methanol. The resulting hydrocarbons were isolated and analyzed by mass-spectrometer. The analysis gives the fraction, $\underline{f}$, of the homo-dimers, masses 238 and 248 , converted into mixed dimers, mass 243 , in a predetermined time interval. The plot of $\ln (1-f)$ vs time was linear and from its slope the first order dissociation constant was calculated, i.e., $\underline{\mathrm{k}}_{\text {diss }}=6.10^{-8} \mathrm{sec}^{-1}$ at $25^{\circ} \mathrm{C}$. In conjunction with the determined association constant, this result gives the equilibrium constant of the dissociation, $\mathrm{k}_{\text {diss }} \sim 10^{-14} \mathrm{M}$. Assuming a plausible value of $15 \mathrm{e} \cdot \mathrm{u}$. for $\Delta S$ of dissociation, one finds the heat of dissociation $\Delta H \sim 24 \mathrm{kcal} / \mathrm{mole}$. 
Another approach led to the dissociation constant of $\mathrm{Na}^{+},-\mathrm{DD}^{-}, \mathrm{Na}^{+}(\operatorname{Ref} .8)$. Since 1,1-diphenyl ethylene does not add to its dimeric dianions, a solution of $\mathrm{Na}^{+},{ }^{-} \mathrm{DD}^{-}, \mathrm{Na}^{+}$was mixed with radioactive 1,1-diphenyl ethylene and the kinetics of exchange was investigated. The results led to the upper limit of the respective dissociation constant, namely $\mathrm{k}^{\prime}$ diss $<10^{-7} \mathrm{sec}^{-1}$.

The stability of the dimeric dianions should be contrasted with the lability of the dimeric radical-anions. For example, indirect evidence reveals that $\mathrm{Na}^{+}, \overline{\mathrm{C}}(\mathrm{Ph})_{2} \mathrm{CH}_{2} \mathrm{CH}_{2} \mathrm{C}(\mathrm{Ph})_{2}$ dissociates in a few microseconds, i.e., its dissociation constant is $10^{5}-10^{6} \mathrm{sec}^{-1}$.

\section{Electron-transfer equilibrium}

Flash-photolysis led also to the determination of electron-transfer equilibrium constants, e.g.,

biphenylide- (B.), $\mathrm{Na}^{+}+1,1$-diphenyl ethylene $(D) \rightleftharpoons$

biphenyl (B) + 1,l-diphenyl ethylene radical-anion: (D:), $\mathrm{Na}^{+}$;

$\mathrm{K}_{\mathrm{tr}}$

This is achieved by flash-photolyzing THF solutions of $\mathrm{Na}^{+},{ }^{-} \mathrm{DD}^{-}, \mathrm{Na}^{+}$in the presence of a mixture of biphenyl and 1,1-diphenyl ethylene of a known composition. Neither hydrocarbon reacts with the dianions, although both were at much higher concentration than $\mathrm{Na}^{+},-\mathrm{D}^{-} \mathrm{D}^{-}, \mathrm{Na}^{+}$. Flash photolysis photo-dissociates some of the dimers into $\mathrm{D}^{-}, \mathrm{Na}^{+}$and then equilibrium

$$
\mathrm{D}^{-}, \mathrm{Na}^{+}+\mathrm{B} \rightleftharpoons \mathrm{D}+\mathrm{B}_{-}^{-}, \mathrm{Na}^{+}, 1 / \mathrm{K}_{\mathrm{tr}},
$$

Is rapidly established due to relatively high concentrations of $B$ and $D$ $\left(\sim 10^{-3} \mathrm{M}\right)$. In the dark period following a flash, the photolyzed dimers are regenerated by the reaction

$$
2 \mathrm{D}^{-}, \mathrm{Na}^{+} \longrightarrow \mathrm{Na}^{+},-\mathrm{DD}^{-}, \mathrm{Na}^{+}, \mathrm{k}_{\mathrm{d}}
$$

Since $\left[D^{-}, \mathrm{Na}^{+}\right]=\mathrm{X} /\left(1+[B] /[D] \mathrm{K}_{t r}\right)$, where $\frac{1}{2} \mathrm{X}$ is the concentration of the dissociated dimers,

$$
-d(1 / X) / d t=k_{d} /\left(1+[B] /[D] k_{t r}\right)^{2}
$$

i.e., plots of the reciprocal of the $\Delta$ optical density at any chosen wavelength are again linear with time. However, their slopes give $\left\{\mathrm{k}_{\mathrm{d}} /\left(I+[\mathrm{B}] /[\mathrm{D}] \mathrm{K}_{\mathrm{tr}}\right) 2\right\} / \mathrm{l}$. In the absence of the added biphenyl, an analogous plot has a slope $\underline{k}_{d} / \ell \varepsilon$, and hence the ratio of both slopes gives $\left(1+[B] /[D] K_{t r}\right)^{2}$. Since [B]/[D] is known, $K_{t r}$ is derived from that experimentally determined ratio.

According to the proposed mechanism, square roots of reciprocals of the slopes should be Iinear with [B] / [D] ratio. This is indeed the case as shown in Fig. 7 . 


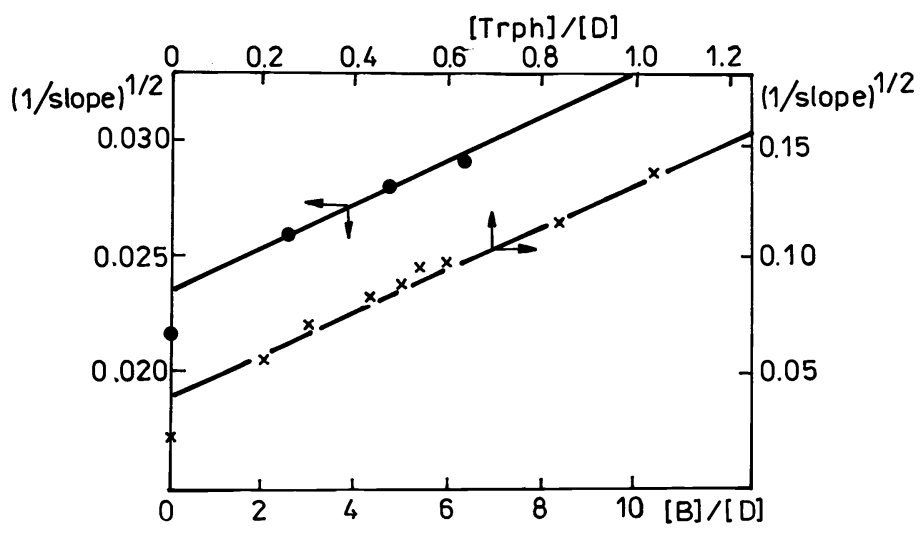

Fig. 7

This approach could not be adopted in the study of the $\propto$-methyl styrene system because $\mathrm{K}^{+},-\propto, \propto, \propto-, \mathrm{K}^{+}$is formed on addition of the monomer to the dimeric dianions, $\mathrm{K}^{+},{ }^{-} \infty \infty^{-}, \mathrm{K}^{+}$. However, addition of an excess of biphenyl, $B$, to a solution of $\mathrm{K}^{+},{ }^{-} \propto \infty^{-}, \mathrm{K}^{+}$changes the character of photolysis, although biphenyl does not react with the dimeric dianions in the dark. In the presence of biphenyl, the transient formed by flash was shown to be its radical-anion, $\mathrm{B}^{-}, \mathrm{K}^{+}$. Nevertheless, all the photolyzed dimeric dianions eventually were regenerated in the dark period, but their formation was substantially slower and obeyed a fourth order kinetics, i.e., the reciprocal of $\Delta$ (optical censity) ${ }^{3}$ was linear with time. This is shown in Fig. 8 .

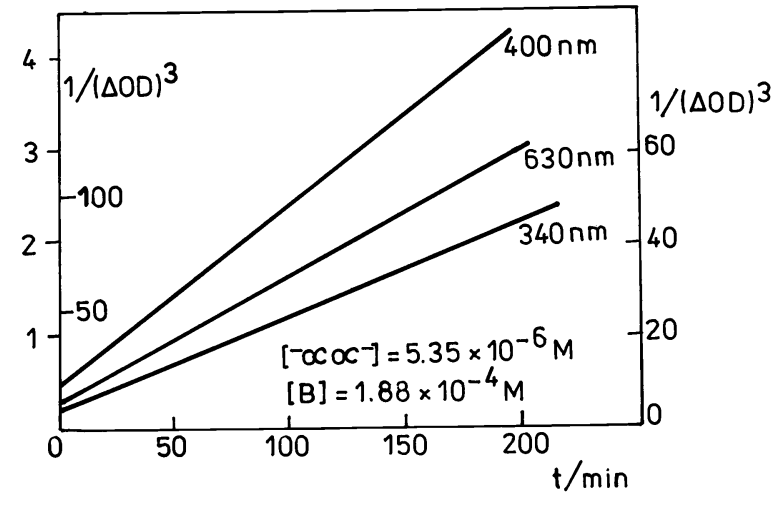

Fig. 8

The basic mechanism discussed in the preceding paragraph accounts for these findings. Virtually all $\alpha^{-}, \mathrm{K}^{+}$is converted into $\mathrm{B}^{-}, \mathrm{K}^{+}$, i.e.,

$$
\propto^{-}, \mathrm{K}^{+}+\mathrm{B}=\propto+\mathrm{B}_{\cdot}^{-}, \mathrm{K}^{+}, \mathrm{K}_{\propto, \mathrm{B}}
$$

with $\propto=\mathrm{B}^{-}, \mathrm{K}^{+}$because $\propto^{-}, \mathrm{K}^{+} \ll \mathrm{B}^{-}, \mathrm{K}^{+}$due to the large excess of $\mathrm{B}^{\text {. Hence, }}$ 


$$
\alpha_{-}^{-}, \mathrm{K}^{+}=\mathrm{X}^{2} / \mathrm{K}_{\mathrm{B}, \infty} \cdot \mathrm{B}
$$

where $\frac{1}{2} X$ is the concentration of the photolyzed dimer. Its regeneration is governed by the equation

$$
-\mathrm{dX} / \mathrm{dt}=\mathrm{k}_{\mathrm{d}} \mathrm{X}^{4} /\left(\mathrm{K}_{\mathrm{B}, c} \cdot \mathrm{B}\right)^{2},
$$

i.e., the plot $1 / \Delta$ (optical density) ${ }^{3}$ is linear with time, its slope being $\frac{1}{3} \underline{k}_{d} /\left(K_{B, \propto} \cdot B\right)^{2}$. Since $\underline{k}_{d}$ is known, $k_{B, \propto}$ could be calculated from the experimentally determined slope.

\section{Other modes of initiation by radical-anions}

Electron-transfer to monomer is not the only mode of initiation caused by radical-anions, although it is unique for non-polar monomers. With some polar monomers, especially cyclic ones, the initiation resembles protonation. For example, the reaction of sodium naphthalenide with ethylene oxide follows the route (Ref. 9)
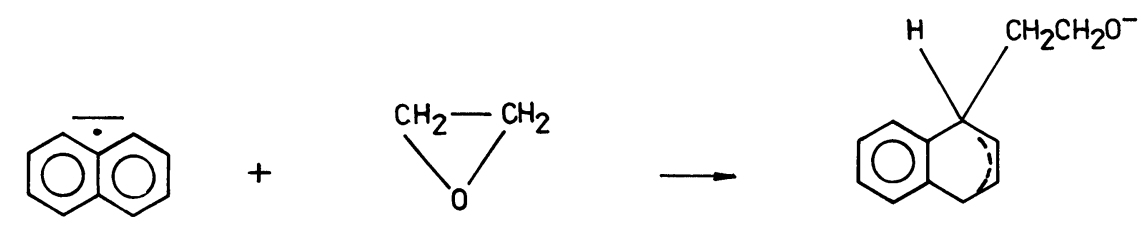

and the reduction of the adduct by the remaining naphthalenide followed by the addition of another molecule of epoxide yields the para or ortho diaduct

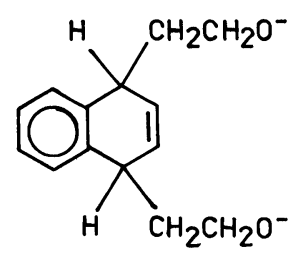

The bimolecular rate constant of the addition of the first molecule of ethylene oxide is $\sim 1 \mathrm{M}^{-1} \mathrm{sec}^{-1}$ (Ref. 10). The subsequent propagation is due to alkoxide ions. A similar process was reported for the initiation of cyclic-tetra-dimethylsiloxane by naphthalenide (Ref. II).

The evidence for this mechanism is two-fold: the preserce of aromatic moiety in the resulting poly-glycol and the quantitative analysis of the solution left after precipitation of the polymer, demonstrating that only one-half of the utilized naphthalenide was converted into naphthalene. ${ }^{x}$

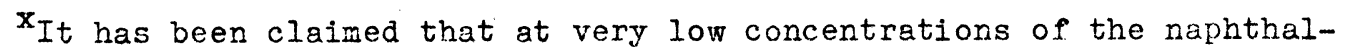
enide, polymers with only one growing group were formed (Ref. 10). Hydrogen abstraction from solvent was proposed as an explanation. However, terminating impurities become significant at very low concentrations of initiatcrs and their action may account for the observations. 
Another variant of initiation by naphthalenide was proposed by Sigwalt (12), who studied the anionic polymerization of propylene sulphide. Formation of propylene in the course of this reaction led him to the following mechanism:

$$
\begin{gathered}
\text { sodium naphthalenide }+ \text { propylene sulphide }- \\
\text { naphatalene }+ \text { NaS. + propylene }
\end{gathered}
$$

NaS. dimerize and the formed sodium disulphide, NaSSNa, inftiates the propagation. The presence of a $\mathbb{S}-\mathbb{S}$ bond in the resulting polymer was claimed in an unpublished report by Boileau and Sigwalt.

\section{Heterogeneous electron-transfer initiation}

Keactions of solid alkali metal particles with monomer or its solutions lead to heterogeneous electron-transfer initiation. The transfer takes place on the surface of the metal to the adsorbed molecules of the monomer and yields adsorbed monomeric radical-anions with the positively charged particles acting as huge counter-ions. Detachment requires not only desorption of the adsorbed radical-anions but also removal of metal cations from the metal lattice.

The hindrance of desorption does not affect the mobility of radicalanions on the metal surface. Hence, their dimerization with formation of still adsorbed dimeric dianions is very likely, and the latter may grow and form living oligomers. The degree of polymerization of the adsorbed oligomers depends on their lifetime on the metal surface, and that lifetime is shortened by a solvent well solvating cations and therefore facilitating the desorption that requires removal of the cation from the metal lattice. This effect is demonstrated by Overberger (13), who studied the co-polymerization of styrene and methyl methacrylate initiated by a fine suspension of metallic lithium particles.

Styrene and methyl methacrylate compete for the sites on the metal surface, the former being favored by the high polarizability of its $\pi$ electrons. Hence, only styrene polymerizes on the surface, yielding a block of living polystyrene. The eventually desorbed living polystyrene initiates polymerization of methyl methacrylate in solution because this monomer is greatly preferred to styrene in anionic polymerization. The greater the hindrance of desorption, the higher the percentage of styrene in the resulting block-polymer. Indeed, as demonstrated by the Overberger study, the size of polystyrene block increased with decreasing solvation power of the medium and the reactions performed in the absence of ethers yields co-polymer with $28 \%$ of styrene at $1 \%$ conversion. On the other hand, the facile removal of $\mathrm{Na}^{+}$cations from the sodium lattice might explain the formation of homo-poly-methyl-methacrylate in similar experiments involving sodium dispersion instead of lithium dispersion.

Further evidence of preferential monomer adsorption on a metal surface is provided by the extensive studies of Richards and his co-workers (14). Alkyl bromides in tetrahydrofuran vigorously react with alkali metals, say lithium, yielding the Wurtz coupling products. However, the violent 
reaction slows down on adition of aromatic monomers like styrene, and the nature of the products is drastically changed (Ref. 15). For example, when an equimolar mixture of ethylbromide and styrene reacts in tetrahydrofuran with metallic lithium, the alkyl capped dimer, $\mathrm{C}_{2} \mathrm{H}_{5} \cdot \mathrm{CH}(\mathrm{Ph})$. $\mathrm{CH}_{2} \cdot \mathrm{CH}_{2} \cdot \mathrm{CH}(\mathrm{Ph}) \cdot \mathrm{C}_{2} \mathrm{H}_{5}$ forms $90 \%$ of the products, about $5 \%$ appear as $\mathrm{C}_{2} \mathrm{H}_{5}$. $\mathrm{CH}_{2} \cdot \mathrm{CH}(\mathrm{Ph}) \cdot \mathrm{C}_{2} \mathrm{H}_{5}$, the remainder being a mixture of an alkyl capped trimer and butane. The tail-to-tail structure of the capped dimer was demonstrated by the n.m.r. technique.

It seems that styrene and ethylbromide compete for the sites on the lithium surface. The adsorption of styrene possessing easily polarizable $\pi$ electrons is more favorable than that of ethylbromide. Hence, Wurtz coupling is hindered, while the electron-transfer to the adsorbed styrene yields its radical-anions, and their mobility on the surface allows for their dimerization. Eventually, the dimeric dianions are desorbed; and since their reaction with ethylbromide is faster than propagation, the ethyl capped dimers are the main products.

Further support for the proposed mechanism is provided by the results of experiments involving phenylbromide instead of ethylbromide (Ref. 16). The polarizable electrons of this aryl compound allow it to compete effectively with styrene for the sites on the lithium surface and thus the viurtz coupling reaction becomes dominant. Similar results were obtained with ethyltosylate. Although the reaction of tosylate with living polystyrene is rapid and quantitative, yielding ethyl capped polymers, its reaction with the monomer and metallic lithium produces only $10 \%$ of the ethyl capped polymers, the remainder being evolved as butane. Again, the aromatic nature of tosylate allows it to compete with styrene for the lithium sites.

An interesting extension of this picture is provided by the behavior of p-xylylene dibromide (Ref. 1?). With butadiene as monomer and tetrahydrofuran as solvent, their reaction on metallic lithium leads to an unusual co-polymer,

$$
-(\mathrm{BD})_{\underline{\mathrm{n}}}-\mathrm{CH}_{2} \cdot \mathrm{C}_{6} \mathrm{H}_{4} \cdot \mathrm{CH}_{2} \cdot \mathrm{CH}_{2} \mathrm{C}_{6} \mathrm{H}_{4} \cdot \mathrm{CH}_{2}-(\mathrm{BD})_{\underline{m}}-\text { (BD-butadiene moiety). }
$$

Its composition is determined by the initial ratio of the reagents in the feed. N.r.r. analysis confirmed the above structure of the aromatic moieties.

$U_{n}$ expectedly, the vicinyl dihalides react differently (Ref. 18). For example, an equimolar mixture of styrene and 1,2-dibromoethane reacts on lithium metal yielding a head-to-head, tail-to-tail polystyrene, ethylene and lithium bromide. Apparently, the adsorbed styrene is reduced and dimerized to the dianions

$$
{ }^{-} \mathrm{CH}(\mathrm{Ph}) \cdot \mathrm{CH}_{2} \cdot \mathrm{CH}_{2} \cdot \mathrm{CH}(\mathrm{Ph})^{-}
$$

and the latter reacts with the dibromide more rapidly than with the unreduced styrene, yielding the unconventional head-to-head, tail-to-tail polymer with elimination of ethylene 


$$
\begin{aligned}
\underline{\mathrm{n}}(\mathrm{FH}(\mathrm{Ph}) & \cdot \mathrm{CH}_{2} \cdot \mathrm{CH}_{2} \mathrm{CH}(\mathrm{Ph})^{-}+-\mathrm{CH}(\mathrm{Ph}) \cdot \mathrm{CH}_{2} \cdot \mathrm{CH}_{2} \mathrm{CH}(\mathrm{Ph})-3 \stackrel{\mathrm{CH}_{2} \mathrm{BrCH}_{2} \mathrm{Br}}{\longrightarrow} \\
& -\left[\mathrm{CH}(\mathrm{Ph}) \cdot \mathrm{CH}_{2} \cdot \mathrm{CH}_{2} \cdot \mathrm{CH}(\mathrm{Ph})-\mathrm{CH}(\mathrm{Ph}) \cdot \mathrm{CH}_{2} \cdot \mathrm{CH}_{2} \cdot \mathrm{CH}(\mathrm{Ph})\right]-\underline{\underline{n} / 2} \\
& +\underline{\underline{n} \mathrm{C}_{2} \mathrm{H}_{4}}+2 \underline{\mathrm{nLiBr}}
\end{aligned}
$$

Other linking agents were investigated, e.g., dibromo-dimethyl-silane and dichlorophenylphosphine (Ref. 19). Interesting products were obtained with di-epoxides, namely

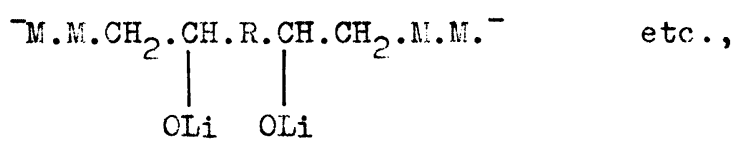

yielding the respective poly-ols on hydrolysis.

\section{Electron-transfer step-wise reaction}

One example of such a reaction is discussed. Reaction of bis(1,1-diphenyl ethylene) linked by a chain of aliphatic hydrocarbon jields on reduction with alkali metals or suitable electron donors, e.g., naphthalenide, a product of poly-dimerization. For example:<smiles>C=C(c1ccccc1)c1ccc(Cc2ccc(C(=C)c3ccccc3)cc2)cc1</smiles><smiles>CC[C+](c1ccccc1)c1ccc(Cc2ccc([C](C[C]I)c3ccccc3)cc2)cc1</smiles><smiles>[CH2]CCc1ccc(Cc2ccc([C](C[CH]C)c3ccccc3)cc2)cc1</smiles>

The resulting poly-carbanions are protonated and yield the respective hydrocarbon (Ref. 20). Other examples are provided by the work of Richards and his co-workers, discussed in the preceding section.

\section{REFERENCES}

1. a) M. Szwarc, Nature 178,1168 (1956); b) M. Szwarc, M. Levy and R. Milkovich, J. Amer. Chem. Soc. 28, 2656 (1956).

2. G. Rämme, M. Fisher, S. Claesson and M. Szwarc, Proc. Roy. Soc. A327, 467 (1972).

3. H.C. Wang, G. Levin and M. Szwarc, J. Phys. Chem. 83, 785 (1979). 
4. a) M. Katayama, M. Hatada, K. Hirota, H. Yamazaki and Y. Ozawa, Bul1. Chem. Soc. Japan 38, 851, 2208 (1965; b) C. Schneider and A.J. Swallow, Hakromolek. Chem. 114, 155, 172 (1968); c) D.J. Metz, R.C. Potter and J.K. Thomas, J. Polymer Sci. A5, 877 (1967).

5. a) H.C. Wang, E.D. Lillie, S. Slomkowski, G. Levin and H. Szwarc, J. Amer. Chem. Soc. 92, 4612 (1977; b) E.D. Lillie, S. Slomkowski and il. Szwarc, J. Amer. Chem. Soc. 29, 4608 (1977).

6. W.H. Hamill, "Radical Ions", p. 321, E.T. Kaiser and I. Kevan, Eds. Interscience (1968).

7. R. Asam1 and M. Szwarc, I. Amer. Chem. Soc. 84, 2269 (1962).

8. G. Spach, M. Monteiro, M. Levy and H. Szwarc, Trans. Faraday Soc. 58, 1809 (1962).

9. D.H. Richards and M. Szwarc, Trans. Faraday Soc. 55, 1654 (1959).

10. S. Kazanskii, A.A. Solovyanov and S.G. Entelis, Europ. Polymer J. 2, 1421 (1971).

11. M. Morton, A. Rembaum and E.E. Bostick, J. Polymer Sci. 32,530 (1958).

12. a) S. Boileau, G. Champetier and P. Sigwalt, J. Polymer Sci. Cl6, 3021 (1967); b) J.P. Favier, S. Boileau and P. Sigwalt, Europ. Polymer J. 4, 3 (1968).

13. C.G. Overberger and N. Yamamoto, J. Polymer Sci. B3, 569 (1965); A4, 3101 (1964).

14. D.H. Richards, Polymer 12, 109 (1978).

15. A. Davis, D.H. Richards and N.F. Scilly, Makromolek. Chem. 152, 121, 133 (1972).

16. A.V. Cunliffe, N.C. Paul, D.N. Richards and D. Thompson, Polymer 19, 329 (1978).

17. D.H. Richards and N.F. Scilly, Brit. Polymer J. 2 , 277 (1970), 3, 101 (1971).

18.D.H. Richards, N.F. Scilly, Chem. Comm., p. 1515 (1968),

19. a) D.H. Richards et 2l., Europ. Polymer J. 6, 1469 (1970);

b) A.V. Cunliffe, W.J. Hubbert and D.H. Richards, Makromolek. Chem. 157, 23, 39 (1972); c) D.H. Richards et al. Polymer 16, 654, 659, 665 (1975).

20. H. Höcker and G. Lattermann, J. Polymer Ści. Symposium 54,361 (1976). 